\title{
Theorie der allgemeinen Periodicität.
}

\author{
Von
}

Otro Raushnberger in Frankfurt a. M.

Die Theorie der transcendenten Functionen kann auf verschiedenen Grundlagen aufgebaut werden. Man kann das bestimmte Integral als Grundelement ansehen und dadurch zu den wichtigeren Transcendenten gelangen, dass man die Integrale algebraischer Ausdrüeke und deren Umkehrungen als neue Functionen einführt. Dieses Verfahren ist bei den elliptischen und in modificirter Weise auch bei den Abel'schen Functionen das historische und konnte in neuerer 'Leit durch die Ausbildung der Theorie der complexen Integrale zu hoher Vollkommenheit gebracht werden. Nichtsdestoweniger haben viele der bedeutendsten Analytiker diesen Weg nicht als den naturgemässen erkannt; wenn derselbe auch $\mathrm{zu}$ allen Eigenschaften der genannten Transcendenten führt, so ist doch nicht zu verkennen, dass er keinen Eínblick in die eigentliche analytische Natur 'derselben gewährt. Andrerseits kann man die unendlichen Reihen und Producte, sowie gewisse Grenzausdrücke zur Definition von Transcendenten benutzen. Der grosse Vorzug dieses Verfahrens liegt darin, dass die genannten Gebilde als Erweiterung der algebraischen Functionen betrachtet werden können und viele Eigenschaften der letzteren auch ihnen zukommen. In der That erscheint die unendliche, für beliebige endliche $x$ convergente Potenzreihe als ganze Function von unendlich hohem Grade; ihre zweite Darstellungsform ist das unendliche Product, zu dem ein nirgends Null werdender Factor hinzutreten kann. Die Erweiterung der rationalen Function ist der Quotient zweier unendlichen Potenzreihen oder Producte; in anderer Form dargestellt giebt sie die unendliche Partialbruchreihe. An diese Gebilde schliessen sich solche unendliche Reihen oder Quotienten derselben an, welche nach Potenzen von rationalen Functionen von $x$ (im einfachsten Fall von $\left.\frac{1}{x}\right)$ fortschreiten. Die verallgemeinerte algebraische Function $y=f(x)$ endlich wird durch eine Gleichung $F(x, y)=0$ definirt, in $\operatorname{der} F(x, y)$ eine unendliche, aus Potenzen von rationalen Functionen der Grössen $x$ und $y$ gebildete Reibe darstellt. 
Das Gebiet der hier skizzirten Functionen ist ein unendlich manuigfaltiges, und es tritt an uns die Frage heran: Nach welchen Principien sollen wir aus dieser zahllosen Menge Transcendenten auswählen, welche so hervorragende Eigenschaften besitzen, dass sie eine besondere Behandlung verdienen? Auf diese Frage ist nach meinem Dafürhalten noch keine befriedigende Antwort gegeben worden; die Einführung der periodischen Functionen geschah entweder durch ziemlich willkürliche Definition oder wurde auf Principien von zu geringer Allgemeinheit gestützt. Die vorliegende und einige später nachfolgende Abhandlungen stellen sich die Aufgabe, in der Beantwortung dieser Frage, wenn vielleicht auch nicht ganz zum Ziele, so doch einen Schritt weiter zu gelangen.

\section{$\S 1$.}

Die periodischen Functionen.

Da die eingeführten Functionen, die wir kurzweg als analytische bezeichnen wollen, nur eine Erweiterung der algebraischen sind, so werden wir behufs einer Auswahl uns nach Analogien in der Theorie der letzteren umsehen müssen. Sei, um den einfachsten Fall zu nehmen, die ganze Function

$$
y=f(x)=a_{0}+a_{1} x+a_{2} x^{2}+\cdots+a_{n} x^{n}
$$

gegeben, so kann zu jedem $x$ das zugehörige $y$ mittelst elementarer Operationen berechnet werden. Dagegen lassen sich, wenn $n>4$ ist, die zu einem gegebenen $y$ gehörigen Werthe von $x$ im Allgemeinen nicht durch Wurzelgrössen u. s. w. ausdrücken, da die definirende Gleichung nicht allgemein algebraisch auflösbar ist. Doch ist bekannt, dass es eine Gruppe höherer Gleichungen giebt, welche eine algebraische Lösung zulassen. Diese sogenannten $\mathrm{A}$ bel'schen Gleichungen sind so beschaffen, dass sich ihre Lösungen in der Form darstellen lassen:

$$
a, \vartheta(a), \vartheta^{2}(a), \cdots, \vartheta^{n-1}(a),
$$

wobei $\vartheta^{\varkappa}$ die $x$ mal iterirte Funetion $\vartheta$ bedeutet und angenommen wird, dass $\vartheta^{n}(a)=a$ ist.

A bel setzt ausserdem voraus, dass $\vartheta$ eine eindeutige Function ist; doch lassen sich jedenfalls auch für mehrdeutige $\vartheta$ ähnliche Betrachtangen durchführen. Durch Anwendung auf algebraische Functionen entspringt hieraus folgender Satż: Eine algebraische Function $y=F(x)$ ist dann algebraisch umkehrbar (d. h. ihre Umkehrung ist durch Wurzelgrössen darstellbar), wenn sie der Functionalgleichung genügt:

aus der weiter folgt:

$$
F(\mathfrak{\vartheta}(x))=F(x),
$$




$$
F(x)=F(\mathfrak{\vartheta}(x))=F\left(\vartheta^{2}(x)\right)=\cdots=F\left(\vartheta^{n-1}(x)\right),
$$

wobei wieder angenommen werden muss, dass $\vartheta^{n}(x)=\vartheta(x)$ ist.

Die Ausdehnung dieser Betrachtung auf transcendente Functionen liegt sehr nahe. Lassen wir die Bedingung fallen, dass $\mathfrak{\vartheta}^{n}(x)=x$ ist, so wird $F(x)$ für unendlich viele verschiedene $x$ denselben Werth annehmen, also keine algebraische function mehr sein können, somit, wenn sie ïberhaupt existirt, eine transcendente Function sein müssen, von der wir wenigstens vermuthen dürfen (die strengere Durchführung muss für später vorbehalten bleiben), dass sich ihre Umkehrung durch eine (im Allgemeinen unendlich grosse) Anzahl von Wurzelgrössen (mit im Allgemeinen unendlich grossen. Exponenten) darstellen lassen wird. Die hervorragende analytische Bedeutung dieser Functionen ist hiernach unmittelbar evident. Wenn nun $F(x)$ der functionalgleichung genügt:

$$
F(\varphi(x))=F(x),
$$

wobei wir uns $y=\varphi(x)$ als eine beliebige algebraische Function, $d$. h. durch eine algebraische Gleichung

$$
\varphi_{1}(x, y)=0
$$

definirt denken wollen, so nennen wir sie eine periodische Function im weiteren Sinn*) oder auch nur kurzweg eine periodische Function, auch wohl zum Uuterschied von später einzuführenden Functionen eine periodische Function erster Gattung. Die gewöhnlich sogenannten periodischen Functionen sind ein specieller Fall der hier definirten; es ist bei ihnen

$$
F(x+n)=F(x)
$$

$\S 2$.

Das algebraische Functionaltheorem.

1. Wir beweisen jetzt einen Satz, welcher die Erweiterung (auch in Bezug auf den Beweis) eines, soviel mir bekannt, von Herrn Weierstrass herrührenden Theorems ist und von einem ganz anderen Gesichtspunkte aus den periodischen Functionen eine hervorragende Wichtigkeit verleiht. Wir fragen: für welche Functionen $f(x)$ besteht ein algebraisches Functionaltheorem derart, dass

$$
f[\psi(x, y)]=\varphi[f(x), f(y)]
$$

ist, wobei $\varphi$ und $\psi$ algebraische, d. h. durch algebraische Gleichungen definirte Functionen bedeuten; oder in Worten ausgesprochen: Wann lässt sich eine Function einer algebraischen Function zweier Variabeln algebraisch ausdrïcken durch die Functionen der einfachen Variabeln?

*) Vgl, hiermit: Comptes rendus, 1879, I, p. 807, Note de M. Appell. 
2. Zunächst ist klar, dass wenn für $f(x)$ jenes Functionaltheorem gültig ist, auch für die Umkehrung desselben, $F(x)$, ein solches stattfinden muss. Denn nehmen wir von beiden Seiten die Function $F$ und setzen statt $x$ und $y F(x)$ und $F(y)$, so wird aus (1):

$$
F[f[\psi(F(x), F(y))]]=F[\varphi[f(F(x)), f(F(y))]],
$$

oder wenn man beachtet, dass

ist,

$$
F[f(x)]=x \quad \text { und } \quad f[F(x)]=x
$$

$$
\psi[F(x), F(y)]=F[\varphi(x, y)] .
$$

In Bezug auf die Vieldentigkeit der vorkommenden Functionen bemerken wir zu dem Vorhergehenden wie zu dem Folgenden, dass wir die betreffenden Gleichungen als befriedigt ansehen, wenn mindestens eine Gruppe der möglichen Werthe denselben genügt.

Beispiel: Für tang $x$ gilt das Functionaltheorem

hieraus folgt:

$$
\operatorname{tang}(x+y)=\frac{\operatorname{tang} x+\operatorname{tang} y}{1-\operatorname{tang} x \cdot \operatorname{tang} y}
$$

$$
\operatorname{arctang} x+\operatorname{arctang} y=\operatorname{arctang} \frac{x+y}{1-x y} .
$$

3. Dass alle algebraischen F'unctionen Gleichungen von der Form (1) genügen, ist unmittelbar evident; es handelt sich nur darum, die transcendenten analytischen Functionen aufzusuchen, welchen diese Eigenschaft zukommt. Dieselben sind alle der Art, dass sie entweder für unendlich viele $x$ denselben Werth $a=f(x)$ annehmen, oder dass dies wenigstens bei ihrer Umkehrung stattfindet. Im letzteren Fall brauchen wir nach dem Vorhergehenden nur ihre Umkehrung zu untersuchen. Seien nun $y_{1}, y_{2}, \cdots, y_{n}$, wobei $n$ die Anzahl der verschiedenen Werthe von $F$ iibersteigen muss, so gewählt, dass

$$
f\left(y_{1}\right)=f\left(y_{\mathfrak{2}}\right)=\cdots=f\left(y_{n}\right)=a
$$

wird, ohne dass jedoch $\psi\left(x, y_{r}\right)=\psi\left(x, y_{s}\right)$ ist, eine Wahl, die immer getroffen werden kann, da wir unendlich viele $\hat{y}$, die der ersten Bedingung genügen, zur Verfügung haben und $\psi(x, y)$ als algebraische Function nur für eine endliche Anzahl der $y$ denselben Werth annimmt. Dann haben wir

$$
\begin{gathered}
\varphi[f(x), a]=f\left[\psi\left(x, y_{1}\right)\right], \\
\varphi[f(x), a]=f\left[\psi\left(x, y_{2}\right)\right], \\
\vdots \\
\varphi[f(x), a]=f\left[\psi\left(x, y_{n}\right)\right] .
\end{gathered}
$$

Da aber die Zahl dieser Gleichungen die Zahl der verschiedenen Werthe von $\varphi[f(x), a]$ übersteigt, so müssen mindestens 2 dieser Grössen übereinstimmen, woraus folgt, dass etwa 


$$
f\left[\psi\left(x, y_{s}\right)\right]=f\left[\psi\left(x, y_{s}\right)\right]
$$

ist. Setzen wir nun aber $x$ statt $\psi\left(x, y_{s}\right)$, so ist $\psi\left(x, y_{r}\right)$ eine algebraische Function $\vartheta(x)$ dieses neuen $x$, und wir erhalten für $f(x)$ die Functionalgleichung:

$$
f[\vartheta(x)]=f(x)
$$

d. h. $f(x)$ ist eine periodische Function. Wir haben somit folgenden Satz bewiesen:

Jede Function, welche ein algebraisches Functionaltheorem der oben definirten Art besitat, ist entweder algebraisch, oder periodisch, oder die Umkehrung einer periodischen Function.

\section{$\S 3$.}

\section{Die Periodicitätsgleichung.}

1. Ist $F\left[\varphi_{1}(x)\right]=F(x)$, so bezeichnen wir $y=\varphi_{1}(x)$ oder auch die entsprechende implicite Gleichung $f(x, y)=0$, welche zwei der Argumente, für die $F(x)$ denselben Werth annimmt, mit einander verbindet, als die zu $F(x)$ gehörige Periodicitätsgleichung. Wir setzen dieselbe in der Folge stets als algebraisch voraus. Bedeutet nun $\varphi_{*}(x)$ die $x$-fach iterirte Function $\varphi_{1}(x), \varphi_{-1}(x)$ die Umkehrung von $\varphi_{1}(x)$ und $\varphi_{-x}(x)$ die $x$-fach iterirte Function $\varphi_{-1}(x)$, so ergeben sich, wenn man an Stelle von $x \varphi_{1}(x), \varphi_{2}(x), \cdots, \varphi_{-1}(x)$ u. s. w. setzt, aus der Gleichung

die folgenden:

$$
F\left[\varphi_{1}(x)\right]=F(x)
$$

$$
\begin{aligned}
F(x) & =F\left[\varphi_{1}(x)\right]=F\left[\varphi_{2}(x)\right]=\cdots \\
& =F\left[\varphi_{-1}(x)\right]=F\left[\varphi_{-2}(x)\right]=\cdots
\end{aligned}
$$

Man ersieht hieraus, dass man $F(x)$ auch die Periode $\varphi_{-1}(x)$ zuschreiben und somit in der Periodicitätsgleichung $f(x, y)=0 x$ mit $y$ vertauschen darf. Dass ausserdem $F(x)$ auch die Periode $\varphi_{x}(x)$ hat, ist ohne Weiteres evident.

2. Setzen wir in $F^{\prime}(x)$ statt $x$ eine algebraische Function dieser Grösse, $\psi_{1}(x)$, so dass wir haben $F_{1}(x)=F\left[\psi_{1}(x)\right]$, und hat wieder $F(x)$ die Periodicitätsgleichung $y=\varphi_{1}(x)$ oder $f(x, y)=0$, so behaupte ich, dass $F_{1}(x)$ ebenfalls periodisch ist und (wenn wir, wie in der Folge, die sich häufenden Klammern theilweise weglassen) die Periodicitätsgleichung $y=\psi_{-1} \varphi_{1} \psi_{1}(x)$ oder in impliciter. Form. $f\left[\psi_{1}(x), \psi_{1}(y)\right]=0$ hat. Setzen wir nämlich $y$ in $F_{1}(x)$ und beachten, dass $\psi_{1} \psi_{-1}(x)=\psi_{-1} \psi_{1}(x)=x$ ist, so erhalten wir oder da

$$
F_{1}\left[\psi_{\rightarrow} \varphi_{1} \psi_{1}(x)\right]=F\left[\psi_{1} \psi_{-1} \varphi_{1} \psi_{1}(x)\right]=F\left[\varphi_{1} \psi_{1}(x)\right]
$$

und

$$
F\left[\varphi_{1}(x)\right]=F(x)
$$


sein muss,

$$
F(x)=F_{1}\left[\psi_{-1}(x)\right]
$$

$$
F_{1}\left[\psi_{-1} \varphi_{1} \psi_{1}(x)\right]=F\left[\psi_{1}(x)\right]=F_{1}(x)
$$

Haben wir daher die Functionen aufgestellt, welchen die Periodicitätsgleichung $y=\varphi_{1}(x)$ oder $f(x, y)=0$ zukommt, so können wir durch die angegebene Functionalsubstitution alle herleiten, welche die Periodicitätsgleichung

$$
y=\psi_{-1} \varphi_{1} \psi_{1}(x) \quad \text { oder } f\left[\psi_{1}(x), \psi_{1}(y)\right]=0
$$

besitzen. Wir werden daher diese doppelte Substitution, welche auch bei anderen, verwandten Untersuchungen von Wichtigkeit ist, anwenden, um verschiedene Arten von Perioden auf gewisse Normalfälle zurückzuführen.

Beispiel: Es ist $\sin (x+2 \pi)=\sin x$, so dass $\sin x$ die Periodicitätsgleichung $y=x+2 \pi$ zukommt. Wählen wir nun $\psi_{1}(x)=x^{2}$, also $\psi_{-1}(x)=\sqrt{x}$, so finden wir, dass $\sin x^{2}$ die Periodicitätsgleichung

$$
y^{2}=x^{2}+2 \pi \text { oder } y=\sqrt{x^{2}+2 \pi}
$$

hat. In der That erhalten wir, wenn wir $y$ statt $x$ in $\sin x^{2}$ einsetzen:

$$
\sin \left[\sqrt{x^{2}+2 \pi}\right]^{2}=\sin \left(x^{2}+2 \pi\right)=\sin x^{2} .
$$

$\S 4$.

Die wiederkehrenden Perioden.

1. Unter den algebraischen Periodicitätsgleichungen $y=\varphi_{1}(x)$ oder $f(x, y)=0$ werden wir zunächst diejenigen auszusuchen haben, welche wiederkehrende Perioden lieferı und in Folge dessen zu keinen transcendenten periodischen Functionen führen, d. h. solche, bei denen $\varphi_{n}(x)=x$ ist. Es handelt sich also darum, alle algebraischen Funetionen aufzufinden, welche $n$ mal iterirt wieder auf $x$ zurückfuhren. Bemerkt muss werden, dass die folgende Untersuchung für mehrdeutige (etwa $x$-dentige) Functionen $\varphi_{1}(x)$ nur insofern gültig ist, als wir blos verlangen, dass einer der $n^{*}$ Werthe, die $\varphi_{n}(x)$ eventuell annehmen kann, gleich $x$ ist.

Eine Function der gesuchten Art lässt sich sofort angeben. Setzen wir nämlich $y=\varphi_{1}(x)=\alpha x$, wobei $\alpha$ eine $n^{\text {te }}$ Einheitswurzel bedeutet, so ist

$$
\varphi_{n}(x)=\alpha^{n} x=x .
$$

Aber auch die Functionen, welche durch Anwendung der Functionalsubstitution aus der eben gefundenen hergeleitet werden können, genügen der Bedingung*); denn ist

*) Die Anwendung der Functionalsubstitution auf $y=\alpha x$, um Functionen der besprochenen Art aufzustellen, findet sich bereits in einem Werke von Hill; doch 
oder kürzer

$$
\varphi_{1}(x)=\psi_{-1}\left[\alpha \cdot \psi_{1}(x)\right]
$$

so haben wir

$$
\varphi_{1}(x)=\psi_{-1} \alpha \psi_{1}(x)
$$

$$
\begin{gathered}
\varphi_{n}(x)=\psi_{-1} \alpha \psi_{1} \psi_{-1} \alpha \psi_{1} \cdots \psi_{-1} \alpha \psi_{1}(x)=\psi_{-1} \alpha^{n} \psi_{1}(x) \\
=\psi_{-1} \psi_{1}(x)=x,
\end{gathered}
$$

wobei auf die oben gemachte Bemerkung uber die Vieldeutigkeit der vorkommenden Functionen Rüeksicht zu nehmen ist.

Ich behaupte nun aber, dass alle möglichen Functionen $\varphi_{1}(x)$ von der gesuchten Art durch die Functionalsubstitution aus $y=a x$ (wobei $\alpha$ alle vorhandenen $n^{\text {len }}$ Einheitswurzeln darstellen mag) hergeleitet werden können. Dies ist bewiesen, wenn ich wirklich eine Substitution angebe, welche das Verlangte leistet. Diese ist aber

$$
\psi_{1}(x)=x+\alpha^{n-1} \varphi_{1}(x)+\alpha^{n-2} \varphi_{2}(x)+\cdots+\alpha \varphi_{n-1}(x)
$$

k'ähren wir nämlich dieselbe in $y=\alpha x$ ein, so wird daraus

oder

$$
\psi_{1}(y)=\alpha \psi_{1}(x)
$$

$$
\begin{gathered}
y+\alpha^{n-1} \varphi_{1}(y)+\alpha^{n-2} \varphi_{2}(y)+\cdots+\alpha \varphi_{n-1}(y) \\
=\alpha\left[x+\alpha^{n-1} \varphi_{1}(x)+\alpha^{n-2} \varphi_{2}(x)+\cdots+\alpha \varphi_{n-1}(x)\right]
\end{gathered}
$$

Eine Lösung dieser Gleichung ist in der That $y=\varphi_{1}(x)$; denn setzen wir darin diese Grösse für $y$ ein, so wird die linke Seite, da $a x^{n}=1$, $\varphi_{n}(x)=x$ ist, $\mathrm{zu}$

$$
\begin{gathered}
\varphi_{1}(x)+\alpha^{n-1} \varphi_{2}(x)+\alpha^{n-2} \varphi_{3}(x)+\cdots+\alpha x \\
=\alpha\left[x+\alpha^{n-1} \varphi_{1}(x)+\alpha^{n-2} \varphi_{2}(x)+\cdots+\alpha \varphi_{n-1}(x)\right],
\end{gathered}
$$

was mit der rechten Seite übereinstimmt.

Diese Substitution wird indessen unbrauchbar, wenn

$$
\psi_{1}(x)=x+\alpha^{n-1} \varphi_{1}(x)+a^{n-2} \varphi_{2}(x)+\cdots+\alpha \varphi_{n-1}(x)
$$

identisch zu einer Constanten $c$ wird. Setzen wir in diesem Ausdruck $\varphi_{1}(x)$ statt $x$, so wird daraus

$$
\varphi_{1}(x)+\alpha^{n-1} \varphi_{2}(x)+\alpha^{n-2} \varphi_{3}(x)+\cdots+\alpha x=\alpha \psi_{1}(x)=\alpha \cdot c ;
$$

fehlt daselbst der Nachweis, dass man dieselben auf diese Weise sämmtlich erhält. Für lineare Functionen ist die allgemeine Untersuchung in verschiedener Weise durchgeführt von Herrn F. Klein, Annalen Bd, IX, pag. 183 und Herrn Gordan, Annalen Bd. XII, pag. 23, von Herrn Camille Jordan in Borchardt's Journal Bd. 84, p. 89. In den genannten Abhandlungen sind die binären Formen mit linearen Transformationen in sich aufgestellt, somit die algebraischen Functionen construirt, welche eine lineare Periodicitätsgleichung besitzen. Es ist biernach gerechtfertigt, wenn die folgenden Untersuchungen auf transcendente Functionen beschränkt werden. - Ausserdem ist zu citiren: Serret, Handbuch der höheren Algebra, deutsche Uebersetzung, 1879, Bd. 2, pag. $299 \mathrm{ff}$. 
es muss demnach $c=\alpha \cdot c$, also $c=0$ sein; $\psi_{1}(x)$ verschwindet dann identisch. In diesem Falle wählen wir die Substitution:

$$
\begin{aligned}
\psi_{1}{ }^{\prime}(x) & =\alpha^{n-1} x \varphi_{1}(x)+\alpha^{n-2} x \varphi_{2}(x)+\cdots+\alpha^{n-k_{1}} \alpha^{n-k_{2}} \varphi_{k_{1}}(x) \varphi_{k_{2}}(x)+\cdots \\
& =\sum_{\substack{k_{1} \\
k_{2}=0,1,2 \ldots n-1 \\
k_{2}<k_{1}}} \alpha^{2 n-k_{1}-k_{2}} \varphi_{k_{1}}(x) \varphi_{k_{2}}(x),
\end{aligned}
$$

und man überzeugt sich leicht wie oben, dass durch dieselbe $y=\varphi_{1}(x)$ aus $y=\alpha^{2} \cdot x$ hergeleitet wird. Falls auch dieser Ausdruck identisch verschwindet (eine von 0 verschiedene Constante kann er wieder nicht sein), so leiten wir $y=\varphi_{1}(x)$ durch die Substitution

$$
\psi_{1}^{\prime \prime}(x)=\sum \alpha^{3 n-k_{1}-k_{2}-k_{3}} \varphi_{k_{1}}(x) \varphi_{k_{2}}(x) \varphi_{k_{3}}(x)
$$

aus $y=\alpha^{3} \cdot x$ her u. s. w.

So können wir weitergehen bis zu

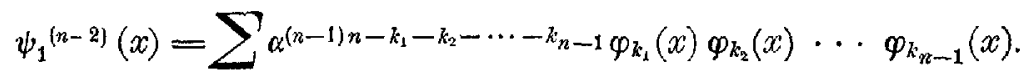

Sollte auch diese Substitution nebst allen vorhergehenden zu Null werden, so wollen wir $\varphi_{1}(x)$ dadurch bestimmen, dass wir eine Gleichung bilden, welche die $n$ Lösungen

$$
x, \alpha^{n-1} \varphi_{1}(x), \alpha^{n-2} \varphi_{2}(x), \cdots \alpha \varphi_{n-1}(x)
$$

besitzt und lautet:

$$
(X-x)\left(X-\alpha^{n-1} \varphi_{1}(x)\right)\left(X-\alpha^{n-2} \varphi_{2}(x)\right) \cdots\left(X-\alpha \varphi_{n-1}(x)\right)=0 \text {. }
$$

Multipliciren wir diese Gleichung aus, so nimmt dieselbe nach bekannten Regeln die Gestalt an:

$$
X^{n}-\psi_{1}(x) X^{n-1}+\psi_{1}^{\prime}(x) X^{n-2}-\cdots \pm \psi_{1}^{(n-2)}(x) X-\Phi(x)=0,
$$

worin $\Phi(x)$ eine unbekannte Function bezeichnet. Da aber voraussetzungsmässig

$$
\psi_{1}(x)=\psi_{1}^{\prime}(x)=\cdots=\psi^{(n-2)}(x)=0
$$

ist, so geht die Gleichung über in

$$
X^{n}-\Phi(x)=0 \text { oder } X=\sqrt[n]{\Phi(x)}=\alpha^{k} \chi(x) .
$$

Geben wir $k$ alle Werthe von 0 bis $n-1$, so müssen wir für $X$ die $n$ Werthe

erhalten. Sei etwa

$$
x, \quad \alpha^{n-1} \varphi_{1}(x), \quad \alpha^{n-2} \varphi_{2}(x), \cdots \alpha \varphi_{(n-1)}(x)
$$

$$
x=\alpha^{r} \chi(x) \text { und } \quad \alpha^{n-1} \varphi_{1}(x)=\alpha^{s} \chi(x),
$$

so folgt daraus durch Elimination von $\chi(x)$

$$
\varphi_{1}(x)=\alpha^{s \cdots n-r+1} x,
$$

d. h. $\varphi_{1}(x)$ hat bereits die gewünschte Form. 
2) In dem besonderen Falle, dass $\varphi_{2}(x)=x$ sein soll, haben wir $\alpha=-1$ zu setzen. Wir erhalten dann die Gleichung

oder

$$
\psi_{1}(y)=-\psi_{1}(x)
$$

$$
\psi_{1}(y)+\psi_{1}(x)=0
$$

d. h. die Periodicitätsgleichung ist in $x$ und $y$ symmetriscb. Dass auch das Umgekehrte gilt, leuchtet sofort ein.

\section{$\S 5$.}

\section{Die rationale Periodicität.}

1. Es handelt sich nunmehr darum, die algebraischen Periodicitätsgleichungen, welche transcendenten Functionen zugehören, mittelst der Functionalsubstitution auf gewisse Normalfulle za reduciren*). Betrachten wir zuerst die allgemeinste in Bezug auf $x$ und $y$ rationale Periodicitätsgleichung

$$
a x y+b x+c y+d=0
$$

in der vorläufig $a$ von 0 verschieden sein möge.

Wir wenden auf dieselbe die Substitution

$$
\psi_{1}(x)=\frac{\alpha+\beta x}{\gamma+\delta x}
$$

an, deren Coefficienten noch unbestimmt und nur der Bedingung unterworfen sein sollen, dass ihre Determinante

$$
\left|\begin{array}{ll}
\alpha & \beta \\
\gamma & \delta
\end{array}\right|=\alpha \delta-\beta \gamma
$$

nicht verschwindet. Wir erhalten dann

$$
a \frac{\alpha+\beta x}{\gamma+\delta x} \cdot \frac{\alpha+\beta y}{\gamma+\delta y}+b \frac{\alpha+\beta x}{\gamma+\delta x}+c \frac{\alpha+\beta y}{\gamma+\delta y}+d=0
$$

oder ausmultiplicirt

$$
\begin{gathered}
{\left[a \beta^{2}+(b+c) \beta \delta+d \delta^{2}\right] x y+[a \alpha \beta+b \beta \gamma+c \alpha \delta+d \gamma \delta] x} \\
+[a \alpha \beta+b \alpha \delta+c \beta \gamma+d \gamma \delta] y+\left[a \alpha^{2}+(b+c) \alpha \gamma+d \gamma^{2}\right]=0 .
\end{gathered}
$$

Man ersieht leicht, dass man unter allen Umständen $\beta$ und $\delta$ so bestimmen kann, dass

$$
a \beta^{2}+(b+c) \beta \delta+a \delta^{2}=0
$$

wird, ohne dass man gleichzeitig $\beta=0$ und $\delta=0$ annehmen müsste. Wir können daher der weiteren Transformation eine einfachere Periodicitätsgleichung zu Grunde legen, welcher das Glied mit $x y$ fehlt:

$$
a x+b y+c=0 \text {. }
$$

*) Die folgende Untersuchung ist, soweit sie sich auf lineare Transformationen bezieht, als bekannt anzuuehmen und soll nur des Zusammenhangs wegen vollständig durchgeführt werden. 
Durch die Substitution

geht dieselbe über in

$$
\psi_{1}(x)=\alpha+\beta x
$$

$$
a \cdot(\alpha+\beta x)+b \cdot(\alpha+\beta y)+c=0 .
$$

Diesen Ausdruck kaun man auf die Normalform

bringen, wenn man

$$
y=p \cdot x
$$

also

und

$$
a \alpha+b \alpha+c=0
$$

$$
\begin{gathered}
\alpha=-\frac{c}{a+b}, \\
b \beta=1, \text { also } \beta=\frac{1}{b}
\end{gathered}
$$

$$
a \beta=p, \text { also } p=\frac{a}{b}
$$

setzt. Diese Reduction wird nur dann unmöglich, wenn $a+b=0$ ist, d. h. wenn die Periodicitätsgleichung in der Form

$$
y=x+n
$$

geschrieben werden kann. -

Hieraus folgt, dass sich alle rationalen Periodicitatsgleichungen auf 2 Normalformen zurückführen lassen:

$$
\begin{aligned}
& \text { 1) } y=p x \\
& \text { 2) } y=x+n
\end{aligned}
$$

2. Wir wollen nun weiter beweisen, dass diese beiden Normalfälle nicht durch eine algebraische Substitution in einander ïbergeführt werden können.

Wäre nämlich $z=\psi_{1}(x)$ eine algebraische Function, welche durch die Gleichung $F(x, z)$, die wix offenbar als irreductibel voraussetzen dürfen, da sie sonst in mehrere zerlegt werden könnte, definirt sein soll, so beschaffen, dass sie als Functionalsubstitution in die Gleichung $y=x+n$ eingesetzt dieselbe in $y=p x$ überführte, so müsste

$$
\psi_{1}(y)=\psi_{1}(x)+n
$$

durch $y=p x$ befriedigt werden, so dass wir hätten:

$$
\psi_{1}(p x)=\psi_{1}(x)+n
$$

Diese Relation kann aber, wenn wir zur definirenden Gleichung $F(x, z)=0$ zuriickgreifen, auch so ausgedrückt werden: die Gleichungen $F(x, z)=0$ und $F(p x, z+n)=0$ sollen für übereinstimmende $x$ durch ein gleiches $z$ befriedigt werden. Da aber voraussetzungsmässig $F(x, z)=0$ irreductibel und $F(p x, z+n)=0$ ron dem nämlichen Grad in $x$ und $z$ sein muss, so folgt, dass $F(x, z)$ und $F(p x, z+n)$ bis auf einen constanten Factor identisch sind. Seien 
nun etwa die höchsten Glieder in Bezug auf $z$ von $F(x, z)$ nach fallenden Potenzen von $x$ geordnet:

$$
z^{k}\left(a_{1} x^{r_{1}}+a_{2} x^{r_{2}}+\cdots\right)+z^{k-1}\left(b_{1} x^{s_{1}}+b_{2} x^{s_{2}}+\cdots\right)+\cdots,
$$

so hätten wir für beliebige $x$ und $z$ die Gleichung $z u$ befriedigen:

$$
\begin{gathered}
(z+n)^{k} \cdot\left(a_{1} p^{r_{1}} x^{r_{1}}+a_{2} p^{r_{2}} x^{r_{2}}+\cdots\right) \\
+(z+n)^{k-1} \cdot\left(b_{1} p^{s_{1}} x^{s_{1}}+b_{2} p^{s_{2}} x^{s_{2}}+\cdots\right)+\cdots \\
=C \cdot\left[z^{k}\left(a_{1} x^{r_{1}}+a_{2} x^{r_{2}}+\cdots\right)+z^{k-1}\left(b_{1} x^{s_{1}}+b_{2} x^{s_{2}}+\cdots\right)+\cdots\right] .
\end{gathered}
$$

Nach Entwickelung der Potenzen von $z+n$ ergiebt aber die Coefficientenvergleichung der ersten Glieder mit $z^{\tilde{k}}$ und $z^{k-1}: a_{1} p^{r_{1}}=C a_{1}$, also, da wir $a_{1}$ als Coefficienten des höchsten Gliedes als von 0 verschieden annehmen müssen,

ferner

$$
C=p^{r_{1}}
$$

$$
\begin{gathered}
k n\left[a_{1} p^{r_{1}} x^{r_{1}}+a_{2} p^{r_{2}} x^{r_{2}}+\cdots\right]+b_{1} p^{s_{1}} x^{s_{1}}+b_{2} p^{s_{2}} x^{s_{2}}+\cdots \\
=C \cdot\left(b_{1} x^{s_{1}}+b_{2} x^{s_{2}}+\cdots\right) .
\end{gathered}
$$

Diese Gleichung kann für beliebige $x$ nur dann statthaben, wenn auf beiden Seiten gleich hohe Potenzen von $x$ vorhanden sind, wenn also etwa $r_{1}=s_{1}$ ist; dann haben wir

oder

$$
k n a_{1} p^{r_{1}}+b_{1} p^{r_{1}}=C \cdot b_{1}
$$

oder

$$
\ln _{n} a_{1} p^{r_{1}}+b_{1} p^{r_{1}}=p^{r_{1}} b_{1}
$$

$$
\eta_{n} n a_{1}+b_{1}=b_{1}
$$

.eine Gleichung, die in keiner Weise zu befriedigen ist, da $k, n, a_{1}$ nothwendig von 0 verschieden sind. Die beiden Normalfälle sind also wesentlich von einander verschieden.

3. Wir haben nun noch zu untersuchen, wie weit sich die beiden Normalperiodicitätsgleichungen in andere derselben Art transformiren lassen. Wenden wir auf $y=x+n$ die Substitution $\psi_{1}(x)=\frac{n}{m} \cdot x$ an, so erhalten wir $y \cdot \frac{n}{m}=x \cdot \frac{n}{m}+n$ oder $y=x+m$, so dass wir jede Function mit einer additiven Periode durch eine lineare Substitution in eine andere derselben Art mit beliebiger anderer Periode verwandeln können.

4. Weniger einfach ist die Untersuchung, wann $\operatorname{sich} y=p x$ in $y=q x$ überführen lässt. Sei wieder $z=\psi_{1}(x)$ eine algebraische Substitution, die das Gewünschte leistet und die durch die irreductible Gleichung $F(x, z)=0$ bestimmt ist. Dann müsste $\psi_{1}(y)=p \psi_{1}(x)$ durch $y=q x$ befriedigt werden, so dass wir hälten $\psi_{1}(q x)=p \psi_{1}(x)$, oder $F(q x, p z)=0$ müsste mit $F(x, z)=0$ eine gemeinschaftliche 
Lösung haben, also wieder, da $F(x, z)=0$ irreductibel ist, mit ihm bis auf eine multiplicatorische Constante übereinstimmen. Sind

$$
a_{1} x^{r_{1}} z^{s_{1}}+a_{2} x^{r_{2}} z^{s_{2}}+\cdots
$$

einige Glieder von $F(x, z)$, so würden wir zu setzen haben

$$
\begin{gathered}
a_{1} q^{r_{1}} p^{s_{1}} x^{r_{1}} z^{s_{1}}+a_{2} q^{r_{2}} p^{s_{2}} x^{r_{2}} z^{s_{2}}+\cdots \\
=C a_{1} x^{r_{1}} z^{s_{1}}+C a_{2} x^{r_{2}} z^{s_{2}}+\cdots
\end{gathered}
$$

und es wäre eine Reihe Gleichnngen von der Form

$$
a q^{r} p^{s}=C a
$$

$\mathrm{zu}$ befriedigen. Sei nun $a$ ron 0 verschiedeu, so ist

$$
C=q^{r} p^{s}
$$

eine andere dieser Gleichungen, etwa

$$
a^{\prime} q^{u} p^{z}=C a^{\prime}
$$

lässt sich nur dann befriedigen ohne $a^{\prime}=0$ zu setzen, wenn $q^{i t} p^{v}=q^{r} p^{s}$ ist. Alle übrigen Glieder müssen nothwendiger Weise verschwinden. Die Gleichung wird daher lauten

oder kürzer

$$
a x^{r} z^{s}+a^{\prime} x^{u} z^{v}=0,
$$

oder

$$
z^{k}=C x^{k_{1}}
$$

$$
z=c x^{\frac{k_{1}}{k}}
$$

wobei $z_{i}$ und $\hbar_{1}$ ganze, positive oder negative Zahlen sind. Wir führen durch diese Substitution $y=p x$ über in $c y^{\frac{k_{1}}{k}}=c p x^{\frac{k_{1}}{k}}$ oder

$$
y=p^{\frac{k}{k_{1}}} x \text {. }
$$

Man kann also $y=p x$ in $y=q x$ nur dann durch eine algebraische Substitution verwandeln, wenn $q$ eine rational gebrochene, positive oder negative Potenz ron $p$ ist.

5. Das Gesammtresultat lautet:

Es giebt nur 2 wesentlich verschiedene Arten von rationalen Perioden: die additive und die multiplicatorische. Für die additiv periodischen Functionen ist die Grösse der Periode nicht charakteristisch, während dies bei den Functionen mit multiplicatorischer Periode der Fall ist. Da die letzteren von einer gewissen constanten Grösse, einem Modul, abhängig sind, so schlage ich für sie den Namen Modularfunctionen vor, der von Gudermann den mit ihnen nahe verwandten elliptischen Functionen beigelegt wurde.

6. Obgleich ich die Construction dieser periodischen Functionen auf eine spätere Gelegenheit verschiebe, so will ich doch folgende Bemerkung über ihre Form beifügen. Ist $f(x+k n)=f(x)$, so wird 
diese Gleichung auch für $k=\infty$ gelten müssen, $\mathrm{d}$. $\mathrm{h}$. eine additiv periodische Function nimmt für $x=\infty$ alle Werthe an, die sie überhaupt für irgend ein $x$ besitzt; $x=\infty$ ist also ein wesentlicher Unstetigkeitspunkt derselben. Hat sie noch einen andern wesentlichen Unstetigkeitspunkt, etwa $x=a$, so müssen wegen der Periodicität auch $x=a+k n$ solche sein, $d$. h. cine additiv periodische Function hat ontweder einen wesentlichen Unstetigkeitspunkt, nämlich $x=\infty$, oder unendlich viele. Im ersten Fall lässt sie sich, wenn sie eindeutig ist, als Potenzreihe von $x$ mit nur positiven Potenzen oder als Qhotient zweier solcher Reihen darstellen.

7. Eine entsprechende Betrachtung ergiebt, dass eine multiplicatorisch periodische Function zwei wesentliche Unstetigheitspunkte bositzt, nämlich $x=\infty$ und $x=0$; hat sie deren noch mëhr, so hat sie unendlich viele. Im ersten Fall muss sie sich (ibre Existenz überhaupt sowie ihre Eindeutigkeit vorausgesetzt) als Potenzreihe mit steigenden und fallenden Potenzen von $x$ oder (was thatsächlich allein möglich ist) als Quotient zweier derartiger Reihen darstellen lassen.

\section{$\S 6$.}

\section{Algebraische Hülfsuntersuchungen.}

Jede Art von Periodicität, welche nicht durch die Gleichung

$$
a x y+b x+c y+d=0
$$

bestimmt ist, muss als irrational und mehrdeutig bezeichnet werden. Denn lautet etwa die Periodicitätsgleichung $y=x^{2}$, so wäre zwar $y$ rational durch $x$, aber nicht $x$ durch $y$ ausgedrückt, und wenn $F\left(x^{2}\right)=F(x)$ ist, so muss auch $F(\sqrt{x})=F(x)$ sein. Bevor wir indessen der Untersuchung dieser irrationalen Perioden näher treten, müssen wir einige allgemeinere Betrachtungen über algebraische Functionen vorausschicken.

1) Sei $y=f(x)$ eine durch die irreductible Gleichung

$$
F(y, x)=0 \text {, }
$$

die in $y$ rom $n^{\text {ten }}$ Grade sein möge, definirte algebraische Function. Es ist bekannt, dass die $n$ Werthe von $y$ im Allgemeinen (einzelne $x$ ausgenommen) von einander verschieden sind, aber dadurch sämmtlich in einander übergeführt werden können, dass man die Variable $x$ einen geschlossenen Weg zurücklegen lässt. Werden nun diese $n$ Werthe von $y$ mit

$$
y_{1}=f^{(1)}(x), \quad y_{2}=f^{(2)}(x), \cdots, y_{n}=f^{(n)}(x)
$$

bezeichnet, so ist einleuchtend, dass sich jeder derselben als algebraische E'unction eines beliebigen andern darstellen lässt; denn man braucht nur aus 


$$
y_{r}=f^{(r)}(x) \quad \text { und } \quad y_{s}=f^{(s)} \dot{(x)}
$$

$x$ zu eliminiren, um eine algebraische Gleichung zwischen $y_{r}$ und $y_{s}$ herzuleiten. Wir nennen diese algebraische Beziehung zwischen je zwei Werthen einer algebraischen Function den Zusammenhang derselben und die Gleichung, welche denselben bestimmt, die Zusammenhangsgleichung.

Sei nun für beliebige $x$

$$
y_{2}=\varphi_{1}\left(y_{1}\right)
$$

so ist klar, dass $\varphi_{1}\left(y_{1}\right)$ auch dann noch eine Lösung von $F(y, x)=0$ darstellen wird, wenn $y_{1}$ durch stetige Aenderung von $x$ in $y_{2}$ übergeführt wird, dass also etwa

$$
y_{3}=\varphi_{1}\left(y_{2}\right)=\varphi_{2}\left(y_{1}\right)
$$

sein muss. Ebenso werden die übrigen Iterirungen von $\varphi_{1}\left(y_{1}\right)$ (d. h. immer je ein Werth dieser im Allgemeinen mohrdeutigen Functionen) Lösungen von $F(y, x)=0$ sein müssen. Da die Anzahl dieser Lösungen eine endliche ist, so muss $p_{1}$ eine Function sein, welche nach einer endlichen Zahl von Iterirungen wieder auf den Ausgangswierth zurückführt, mithin sich nach $\S 4$. in der Form

$$
\varphi_{1}\left(y_{1}\right)=\psi_{-1} \alpha \psi_{1}\left(y_{1}\right)
$$

darstellen lassen $\left(\psi_{1}\right.$ ist eine algebraische Function, $\alpha$ eine $k^{\text {le }}$ Einheitswurzel). - Haben wir nun

so wird auch etwa

$$
\begin{gathered}
y_{2}=\varphi_{1}\left(y_{1}\right), \\
y_{3}=\varphi_{2}\left(y_{1}\right), \\
\vdots \\
y_{k}=\varphi_{k-1}\left(y_{1}\right), \\
y_{1}=\varphi_{k}\left(y_{1}\right),
\end{gathered}
$$

$$
\begin{gathered}
y_{k+2}=\varphi_{1}\left(y_{k+1}\right), \\
y_{k+3}=\varphi_{2}\left(y_{k+1}\right), \\
\vdots \\
y_{2 k}=\varphi_{k-1}\left(y_{k+1}\right), \\
y_{k+1}=\varphi_{k}\left(y_{k+1}\right)
\end{gathered}
$$

sein und kein $y$ der zweiten Reihe wird mit einem der ersten übereinstimmen können. Durch Fortsetzung dieses Verfahrens lassen sich sämmtliche $n$ Werthe von $y$ in Reihen von je $k$ Gliedern ordnen, woraus man erkennt, dass $\hbar$ ein Theiler von $n$ sein muss. Ist ferner

$$
y_{k+1}=x_{1}\left(y_{1}\right)
$$

so wird $\chi_{l}\left(y_{1}\right)=y_{1}$ und $l$ auch ein Theiler von $n$ sein müssen u. s. w. Auf diese Weise können sich noch mehrere Reihen von Zusammenhangsgleichungen zwischen den Lösungen ergeben, falls $n$ sich in 
Factoren zerlegen lässt; ist dagegen $n$ eine Primzahl, so muss der Zusammenhang ein einfacher sein, d. h. alle Lösungen lassen sich aus einer einzigen durch Iterirung einer Function herleiten. - Als primitiv wollen wir die Zusammenhangsgleichung

$$
y_{2}=\varphi_{1}\left(y_{1}\right)
$$

bezeichnen, wenn $\varphi_{1}\left(y_{1}\right)$ nicht die Iterirung einer andern Function $\chi_{1}(y)$ ist, welche durch ihre Iterirungen eine grössere Anzahl von Functionalwerthen liefert wie $\varphi_{1}\left(y_{1}\right)$. - Wir sprechen von einem einfachen, doppelten, dreifacheu u. s. w. Zusammenhang einer algebraischen Function, wenn sich alle Werthe derselben durch Iterirung einer, zweier, dreier u. s. w. primitiver Zusammenhangsfunctionen aus einer einzigen herleiten lassen.

Einige Beispiele werden die Sache deutlicher machen.

Beispiel 1:

Wir haben

$$
\begin{aligned}
& (y-1)^{4}=x \\
& y_{1}=1+\sqrt[4]{x} \\
& y_{2}=1+i \sqrt[4]{x} \\
& y_{3}=1-\sqrt[4]{x} \\
& y_{4}=1-i \sqrt[4]{x}
\end{aligned}
$$

Die Zusammenhangsgleichung zwischen $y_{2}$ und $y_{1}$ lautet:

oder

$$
y_{2}-1=i\left(y_{1}-1\right) \text {, }
$$

$$
\varphi_{1}\left(y_{1}\right)=y_{2}=i y_{1}+1-i \text {. }
$$

Dieselbe ist primitiv, denn wir haben

also

$$
\varphi_{2}\left(y_{1}\right)=-y_{1}+2 \text {, }
$$

und

$$
y_{3}=\varphi_{2}\left(y_{1}\right),
$$

also

Dagegen ist

$$
\varphi_{3}\left(y_{1}\right)=-i y_{1}+1+i,
$$

$$
y_{4}=\varphi_{3}\left(y_{1}\right) \text {. }
$$

$$
\psi_{1}\left(y_{1}\right)=\varphi_{2}\left(y_{1}\right)=-y_{1}+2
$$

nicht primitiv, da schon

ist.

$$
\psi_{2}\left(y_{1}\right)=y_{1}
$$

Beispiel 2:

also

$$
y^{2}-2 y x+x^{2}-x=0,
$$

$$
\begin{aligned}
& y_{1}=x+\sqrt{x} \\
& y_{2}=x-\sqrt{x}
\end{aligned}
$$


und somit

$$
\varphi_{1}\left(y_{1}\right)=y_{2}=1+y_{1} \pm \sqrt{1+4 y_{1}}
$$

setzt man hierin $y_{2}$ statt $y_{1}$, so folgt

$$
\varphi_{2}\left(y_{1}\right)=2+y_{1} \pm \sqrt{1+4 y_{1}} \pm\left(2 \pm \sqrt{1}+4 y_{1}\right)
$$

worin nur das erste und dritte Doppelzeichen correspondiren. Die Werthe ron $\varphi_{2}\left(y_{1}\right)$ sind also

$$
4+y_{1} \pm 2 \sqrt{1+4 y_{1}} \text { und } y_{1}
$$

zwei Werthe geben also wieder die erste Lösung.

Beispiel 3:

oder

$$
y^{4}-2 y^{2}=x
$$

$$
\begin{aligned}
& y_{1}=+\sqrt{1+\sqrt{1+x}} \\
& y_{2}=-\sqrt{1+\sqrt{1+x}} \\
& y_{3}=+\sqrt{1-\sqrt{1+x}} \\
& y_{4}=-\sqrt{1-\sqrt{1+x}} .
\end{aligned}
$$

Hier ist der Zusammenhang ein doppelter; denu wir haben

und

$$
y_{1}+y_{2}=0
$$

$$
y_{1}^{2}+y_{3}^{2}=2 \text {. }
$$

2. Die allgemeine Darstellung der Zusammenhangsgleichungen einer durch eine algebraische Gleichung definirten Function ist sehr leicht. Sei

durch

$$
y=f(x)
$$

$$
\begin{gathered}
F(y, x)=y^{n}+\chi_{1}(x) y^{n-1}+\chi_{2}(x) y^{n-2}+\cdots+\chi_{n-1}(x) y \\
+\chi_{n}(x)=0
\end{gathered}
$$

bestimmt, worin die $\chi(x)$ rationale Functionen von $x$ bedeuten, so brauchen wir nur zwischen

und

$$
F\left(y_{r}, x\right)=0
$$

$$
F\left(y_{s}, x\right)=0
$$

$x$ zu eliminiren. Etwas umständlicher, doch für die Theorie wichtig ist folgende Methode. Wir haben

$$
\begin{gathered}
y_{1}+y_{2}+\cdots+y_{n}=-x_{1}(x) \\
y_{1} y_{2}+y_{1} y_{3}+\cdots+y_{n-1} y_{n}=x_{2}(x) \\
\vdots \\
y_{1} y_{2} \cdots y_{n}=(-1)^{n} x_{n}(x)
\end{gathered}
$$


Eliminirt man aus diesen $n$ Gleichungen $x$ und $n-2$ der $y$, so erhält man eine Gleichung zwischen den beiden übrigen $y$. Bei beiden Methoden ist ersichtlich, dass das Eliminationsresultat genau gleich ausfallen muss, welche beiden $y$ man auch in die Zusammenhangsgleichung bringt. Die gefundene Gleichung umfasst daher den Zusammenhang aller Paare von Werthen mit Einschluss von $y_{r}=y_{s}$ und ist somit nicht irreductibel. - Bei dem zuletzt betrachteten Beispiel

ergiebt sich

$$
y^{4}-2 y^{2}=x
$$

oder

$$
y_{r}^{4}-2 y_{r}^{2}=y_{s}^{4}-2 y_{3}^{2}
$$

$$
y_{r}{ }^{4}-y_{s}{ }^{4}-2 y_{r}{ }^{2}+2 y_{s}{ }^{2}=0 \text {, }
$$

eine Gleichung, die sich in die 3 Factoren

zerlegen lässt.

$$
\begin{aligned}
& y_{r}-y_{s}=0, \\
& y_{r}+y_{s}=0, \\
& y_{r}{ }^{2}+y_{s}{ }^{2}-2=0
\end{aligned}
$$

3. Setzen wir in der Gleichung $F(x, y)=0$ mit den Lösungen $y_{k}=f^{(k)}(x)$

$$
y=\xi_{1}(z)=\frac{a z+b}{c z+d}
$$

so sind die Lösungen der transformirten Gleichung

$$
z_{k}=\xi_{-1} f^{(k)}(x) \text {, }
$$

und wenn eine Zusammenhangsgleichung von $F(y, x)=0$ lautete:

oder

$$
\Phi\left(y_{r}, y_{s}\right)=0 \text {, }
$$

so haben wir jetzt

$$
y_{s}=\Psi_{1}\left(y_{r}\right)
$$

oder

$$
\Phi\left(\xi_{1}\left(z_{r}\right), \xi_{1}\left(z_{s}\right)\right)=0
$$

$$
z_{s}=\xi_{-1} \Psi_{1} \xi_{1}\left(z_{r}\right)
$$

Die Zusammenhangsgleichungen gestatten also dieselbe Transformation wie die Periodicitätsgleichungen, doch nur mittelst einer linearen Substitation.

4. Von besonderer Wichtigkeit sind für uns diejenigen Functionen $y=f_{1}(x)$, welche durch eine Gleichung von der Form

$$
\frac{a_{0} y^{n}+a_{1} y^{n-1}+\cdots+a_{n}}{b_{0} y^{n}+b_{1} y^{n-1}+\cdots+b_{n}}=\varphi_{1}(x),
$$

oder, was dasselbe ist,

(2) $y^{n}+\frac{a_{1}-b_{1} \varphi_{1}(x)}{a_{0}-b_{0} \varphi_{1}(x)} y^{n-1}+\frac{a_{2}-b_{2} \varphi_{1}(x)}{a_{0}-b_{0} \varphi_{1}(x)} y^{n-2}+\cdots+\frac{a_{n}-b_{n} \varphi_{1}(x)}{a_{0}-b_{0} \varphi_{1}(x)}=0$ 
bestimmt sind, worin $\varphi_{1}(x)$ eine rationale l'unction bedeutet. Die Zusammenhangsgleichung von (1) oder (2) lautet

$$
\frac{a_{0} y_{r}^{n}+a_{1} y_{r}^{n-1}+\cdots+a_{n}}{b_{0} y_{r}^{n}+b_{1} y_{r}^{n-1}+\cdots+b_{n}}=\frac{a_{0} y_{s}^{n}+a_{1} y_{s}^{n-1}+\cdots+a_{n}}{b_{0} y_{s}^{n}+b_{1} y_{s}^{n-1}+\cdots+b_{n}},
$$

ist also von $\varphi_{1}(x)$ unabhängig. Dividirt man (3) durch den Factor $y_{r}-y_{s}$, so liefert die übrigbleibende Gleichung $(n-1)^{\text {tn }}$ Grades genau die $n-1$ Werthe von $f_{\mathrm{t}}(x)$, welche ausser $y_{r}$ noch existiren. Es ist aber auch leicht zu erweisen, dass jede $n$ deutige algebraische Function, welche vollständig denselben Zusammenhang wie die durch (1) definirte hat, von der Form

$$
\frac{a_{0} y^{n}+a_{1} y^{n-1}+\cdots+a_{n}}{b_{0} y^{n}+b_{1} y^{n-1}+\cdots+b_{n}}=\varphi_{1}^{\prime}(x)
$$

sein muss. Denn soll

$$
y^{n}+\chi_{1}(x) y^{n-1}+\chi_{2}(x) y^{n-2}+\cdots+\chi_{n}(x)=0
$$

die verlangte Eigenschaft haben, so müssen alle Gleichungen, welche sich zwischen den Lösungen ron (1) oder $(2): y_{1}, y_{2}, \cdots, y_{n}$ aufstellen lassen, identisch mit denjenigen sein, welche sich zwischen den Lösungen von (5): $y_{1}^{\prime}, y_{2}^{\prime}, \cdots, y_{n}^{\prime}$ ergeben. Da wir aber

und

$$
\left\{\begin{array}{c}
y_{1}+y_{2}+\cdots+y_{4}=-\frac{a_{1}-b_{1} \varphi_{1}(x)}{a_{0}-\frac{b_{0} \varphi_{1}(x)}{y_{1}}} \\
y_{1} y_{2}+y_{1} y_{3}+\cdots+y_{n-1} y_{n}=\frac{a_{2}-b_{2} \varphi_{1}(x)}{a_{2}-b_{0} \varphi_{1}(x)} \\
\vdots \\
y_{1} y_{2} \cdots y_{n}=(-1)^{n} \frac{a_{n}-b_{n} \varphi_{1}(x)}{a_{0}-b_{0} \varphi_{1}(x)}
\end{array}\right.
$$

$$
\left\{\begin{array}{c}
y_{1}^{\prime}+y_{2}^{\prime}+\cdots+y_{n}^{\prime}=-\chi_{1}(x), \\
y_{1}^{\prime} y_{2}^{\prime}+y_{1}^{\prime} y_{3}^{\prime}+\cdots+y_{n-1}^{\prime} y_{n}^{\prime}=\chi_{2}(x), \\
\vdots \\
y_{1}^{\prime} y_{2}^{\prime} \cdots y_{n}^{\prime}=(-1)^{n} \chi_{n}(x)
\end{array}\right.
$$

haben, so werden die Resultate der Elimination von $x$ zwischen

und

$$
\sum y_{1} y_{2} \cdots y_{r}=(-1)^{r} \frac{a_{r}-b_{r} \varphi_{1}(x)}{a_{0}-b_{0} \varphi_{1}(x)}
$$

$$
\sum y_{1} y_{2} \cdots y_{s}=(-1)^{s} \frac{a_{s}-b_{s} \varphi_{1}(x)}{a_{0}-\frac{b_{0} \varphi_{1}(x)}{2}}
$$

oder, was auf dasselbe hinausläuft,

und

$$
\sum y_{1} y_{2} \cdots y_{r}=(-1)^{r} \frac{a_{r}-b_{r} x}{a_{0}-b_{0} x}
$$


einerseits und

$$
\sum y_{1} y_{2} \cdots y_{s}=(-1)^{s} \frac{a_{s}-b_{s} x}{a_{0}-b_{b} x}
$$

$$
\sum y_{1}^{\prime} y_{2}^{\prime} \cdots y_{r}^{\prime}=(-1)^{r} \chi_{r}(x)
$$

und

$$
\sum y_{1}^{\prime} y_{2}^{\prime} \cdots y_{s}^{\prime}=(-1)^{s} x_{s}(x)
$$

andrerseits identisch sein müssen. Es ist aber leicht einzusehen (was weiter unten bei anderer Gelegenheit ausführlicher erörtert werden wird), dass dies nur dann zutrifft, wenn

und

$$
\chi_{r}(x)=\frac{a_{r}-b_{r} \varphi_{i}^{\prime}(x)}{a_{0}-b_{0} \varphi_{1}^{\prime}(x)}
$$

$$
\chi_{s}(x)=\frac{a_{s}-b_{3} \varphi_{1}^{\prime}(x)}{a_{0}-b_{0} \varphi_{1}^{\prime}(x)}
$$

ist, worin $\varphi_{1}^{\prime}(x)$ nur eine rationale Function sein kann, weil sonșt die Coefficienten der Gleichung vieldeutig würden und hierdurch der Züsammenhang eine Aendexung erlitte. Hiermit ist die Behauptung erwiesen. -

Mit diesen wenigen Resnltaten ist natürlich die Theorie des $\mathrm{Zu}-$ sammenhangs nicht erschöpft; doch genügen dieselben, um die Untersuchungen über irrationale Periodicität bis zu einem gewissen Punkte zu erledigen.

\section{$\S 7$.}

\section{Dio irrationale Periodicität.}

1. Um die recht schwierige Frage über die Zulässigkeit der jrrationalen Periodicität klarer zu machen, will ich mich vorerst an ein Beispiel halten. Wenn die Periodicitätsgleichung der eindeutigen Function $F(x)$ lautet:

$$
y=x^{2}
$$

so müssen die Relationen bestehen:

$$
\begin{aligned}
F(x) & =F\left(x^{2}\right)=F\left(x^{4}\right)=F\left(x^{8}\right)=\cdots \\
& =F(\sqrt{x})=F(\sqrt{x})=F(\sqrt[8]{x})=\cdots
\end{aligned}
$$

$\sqrt{x}$ hat aber zwei Werthe, welche wir als $+\sqrt{x}$ und $-\sqrt{x}$ unterscheiden könneu. Es entsteht nun die Frage: sollen wir ron der periodisehen Function $F(x)$ verlangen, dass sowohl $F(+\sqrt{x})$ als auch $F(-\sqrt{x})$ mit $F(x)$ übereinstimmt, oder sollen wir es als genügend betrachten, wenn nur eine dieser Grössen, etwa $F(+\sqrt{x})$ gleich $F(x)$ wird? Nehmell wir für einen Augenblick das Letztere an, setzen aber voraus, dass $F(x)$ nur in einzelnen Punkten unstetig wird. Sei nun 
$F\left(+\sqrt{x_{1}}\right)=F\left(x_{1}\right)$ und denken wir uns, dass $x$ von $x_{1}$ eine continuirliche Reihe von Werthen durchläuft, ohne jedoch durch einen Unstetigkeitspunkt von $F(x)$ oder $F(+\sqrt{x})$ zu gehen, was nach der eben gemachten Voraussetzung immer möglich ist; so wird, da $F(x)$ und $F(\sqrt{x})$ nicht plötzlich ihren Werth ändern können, diese Uebereinstimmung fortwälrend stattfinden müssen. Nun ist es aber bekanntlich immer möglich (und zwar auf unendlich vielen Wegen), die Variable so zu führen, dass, wenn $x$ zu $x_{1}$ zurückkehrt, $+\sqrt{x_{1}}$ in $-\sqrt{x_{1}}$ übergegangen ist. Demnach müsste anch $F(-\sqrt{x})=F(x)$ sein. Man übersieht sofort, dass, wenn eine für beliebige $x$ (einzelne Punkte ausgenommen) stetige Function eine Periode $y=\varphi_{1}(x)$ haben soll, welche durch eine irreductible Gleichung definirt ist (was immer vorausgesetzt werden darf, da sich sonst die Gleichung in mehrere zerlegen liesse), die Gleichungen

$$
F\left(\varphi_{x}(x)\right)=F(x) \text { und } F\left(\varphi_{-x}(x)\right)=F(x)
$$

für sämmtliche Werthe von $\varphi_{*}(x)$ und $\varphi_{-x}(x)$ bestehen müssen. Kehren wir jedoch zu unserem Beispiel zurück. Es müsste $F(\sqrt{x})$ für die 2 Werthe von $V \bar{x}, F(\sqrt[2]{x})$ für die $2^{*}$ Werthe von $\sqrt[2^{x}]{x}$ mit $F(x)$ übereinstimmen. Allein wir könuten ja auch $F\left(x^{2}\right)=F(x)$ zum Ausgangspunkt nehmen; dann müsste $F\left( \pm \sqrt{x^{2}}\right)=F(x)$ oder $F( \pm x)=F(x)$, ferner $F\left( \pm \alpha^{2^{x}} \sqrt{x}\right)=F(x)$ sein, wenn $\alpha$ eine $2^{x \text { te }}$ Einheitswurzel bedeutet. Hierbei würden wir also noch mehr Werthe des Arguments finden, für welcbe $F(x)$ sich gleich bleiben soll. Wenn man $F\left(x^{4}\right)$, $F\left(x^{8}\right)$ u. s. w. zum Ausgangspunkt wählt, so mehrt sich noch die Zahl dieser Werthe, so dass $F(x)$ für $2^{\infty}$ Werthe sich gleich bleiben müsste. Aehnliches tritt im Allgemeinen bei mehrdeutigen Periodicitätsfunctionen ein. Da es nun wahrscheinlich, wenn auch nicht erwiesen ist, dass eine stetige Function nur für $\infty^{2}$ Werthe des Arguments unverändert bleiben kann, so schliessen wir die Functionen mit mehrdeutiger Periodicitätsbeziehung im. Allgemeinen von dem Kreis der zu untersuchenden aus.

2. Bemerkt zu werden verdient, dass diese nicht 'ganz exacten Betrachtungen in speciellen Fällen mit völliger Strenge durchgeführt werden können. Ist z. B. $y=\varphi_{1}(x)=x^{n}$, so ergiebt sich leicht, dass $F(\alpha x)=F(x)$ sein muss, wenn $\alpha$ eine $n^{x \text { te }}$ Einheitswurzel bedeutet. Die Reihe der $n^{x \text { ten }}$ Einheitswurzeln stellt aber für ein sehr grosses $x$ einen geschlossenen Kreis von unendlich benachbarten Werthen dar, sodass wegen der vorausgesetzten Stetigkeit von $F(x)$ diese Function für eine zusammenhängende Reihe von Werthen, also überhaupt unverändert bleiben müsste. 
3. Die in 1. gemachten Schlüsse gelten nur so lange, als $F(x)$ eine eindeutige Function ist; schon bei endlich vieldentigen functionen verlieren sie ihre Gültigkeit, wie das Beispiel

$$
F(x)=\sin \sqrt[3]{x}
$$

zeigt, welches eine dreidentige Funetion mit den Werthen $\left(\alpha^{3}=1\right)$

$$
\begin{aligned}
& F^{(1)}(x)=\sin \sqrt[3]{x}, \\
& F^{(2)}(x)=\sin \alpha \sqrt[3]{x} \\
& F^{(3)}(x)=\sin \alpha^{2} \sqrt[3]{x}
\end{aligned}
$$

und der Periodicitätsgleichung

oder

$$
\sqrt[3]{y}=2 \pi+\sqrt[3]{x}
$$

$$
y=(2 \pi+\sqrt[3]{x})^{3}
$$

repräsentirt. Setzt man nämlich $y$ statt $x$ in $F(x)$, so erhält man die 9 Ausdrücke

$$
\begin{array}{rrr}
\sin (2 \pi+\sqrt[3]{x}), & \sin (2 \pi+\alpha \sqrt[3]{x}), & \sin \left(2 \pi+\alpha^{2} \sqrt[3]{x}\right), \\
\sin \alpha(2 \pi+\sqrt[3]{x}), & \sin \alpha(2 \pi+\alpha \sqrt[3]{x}), & \sin \alpha\left(2 \pi+\alpha^{2} \sqrt[3]{x}\right), \\
\sin \alpha^{2}(2 \pi+\sqrt[3]{x}), & \sin \alpha^{2}(2 \pi+\alpha \sqrt{x}), & \sin \alpha^{2}\left(2 \pi+\alpha^{2} \sqrt[3]{x}\right),
\end{array}
$$

von denen der erste, zweite und dritte bezüglich $\sin \sqrt{x}, \sin \alpha \sqrt[3]{x}$, $\sin \alpha^{2} \sqrt[3]{x}$ gleich sind, während die übrigen andere Werthe annehmen. Auch ist es nicht möglich, derartige mehrdeutige Functionen mit irrationaler Periode auf eindeutige zurückzuführen, indem man sie als Lösungen einer algebraischen Gleichung darstellt, deren Coefficienten eindentige periodische Functionen sind. Der eindeutige Ausdruck .

$$
\begin{gathered}
\sin \sqrt[3]{x}+\sin \alpha \sqrt[3]{x}+\sin \alpha^{2} \sqrt[3]{x}= \\
-\frac{x}{1 \cdot 2}+\frac{x^{3}}{1 \cdot 2 \cdot 3 \cdot 3 \cdot 3}-\frac{x^{5}}{1 \cdot 2 \cdot 3 \cdots 14 \cdot 5}+\cdots
\end{gathered}
$$

ist überhaupt nicht periodisch, da die einzelnen Theile desselben nicht bei gleichen Aenderungen ungeändert bleiben. - Dass unendlich vieldeutige Functionen irrationale Periodicitätsgleichungen zulassen, die bei eindeutigen und endlich vieldeutigen Functionen unmöglich sind, zeigt folgende Function:

$$
F(x)=\sin \left(2 \pi \frac{\log \log x}{\log 2}\right)
$$

diese bleibt ungeändext, wenn n!an $x^{2}$ oder $x^{\frac{1}{2}}$ statt $x$ setat; nur ist letzteres nicht für beide Werthe ron $x^{\frac{1}{2}}$ der Fall. - Im Folgenden beschränken wir uns auf die Untersuchung eindeutiger Functionen. 
4. Die Schwierigkeiten, auf welche die irrationale Periodicität führt, sind in 2 Fällen nicht vorbanden:

a) wenn die Periodicitätsfunction derart ist, dass keine Iterirung derselben mehr als eine bestimmte, endliche Anzahl von Werthen besitzt;

b) wenn die Zahl der Werthe von $\varphi_{n}(x)$ zwar ins Beliebige wächst, allein unter denselben nur eine bestimmte, endliche Zahl von den Werthen früherer lterirungen verschieden ist.

5. Der erste Fall, welchen ich allein erledigt habe, lässt sich leicht reduciren. Es ist vorerst klar, dass keine der Iterirungen der $n$-cleutigen, durch eine irreductible Gleichung definirten Function $y=f_{1}(x)$ weniger als $n$-deutig sein kann. Denn seien $y_{1}, y_{2}, \cdots, y_{r}$ die Werthe von $f_{x-1}(x)$, so haben wir für $f_{x}(x)$ :

$$
f_{1}\left(y_{1}\right), f_{1}\left(y_{2}\right) \cdots f_{1}\left(y_{r}\right) \text {. }
$$

Jeder dieser Ausdrücke ist $n$-werthig, da $f_{1}(x)$ für dasselbe Argument $n$ wesentlich verschiedene Werthe (einzelne Punkte ausgenommen) haben muss; die Gesammtzahl der Werthe von $f_{x}(x)$ muss daher mindestens $n$ betragen. Nehmen wir nun an, dass die Vieldeutigkeit der Iterirungen von $f_{1}(x)$ bis zu einer $s$-fachen steige, und dass $f_{x}(x)$ dieselbe erreiche, so werden auch $f_{2 x}(x), f_{3 x}(x) u$.s. w. gerade $s$-deutig sein, weil ein höherer Grad der Vieldeutigkeit nach der gemachten Annahme, ein niedrigerer aber nach dem eben Bewiesenen nicht möglich ist. Da nun eine Function nit der Periode $y=f_{1}(x)$ auch die Periode $y=f_{x}(x)$ hat, so genügt es, diejenigen Periodicitätsfunctionen $y=f_{1}(x)$ aufzusuchen, deren Iterirungen von derselben $\nabla$ ieldeutigkeit wie $f_{1}(x)$ sind.

6. Soll dies bei der ersten Iterirung der $n$-deutigen Function $f_{1}(x)$ der Fall sein, so werden, wenn $y_{1}, y_{2}, \ldots, y_{n}$ die Werthe ron $f_{1}(x)$ bezeichnen,

$$
f_{1}\left(y_{1}\right), f_{1}\left(y_{2}\right), \cdots, f_{1}\left(y_{n}\right)
$$

dieselben Werthe liefern müssen, da jeder dieser Ausdrücke deren $n$ besitzt und im Ganzen nur $n$ vorhanden sein sollen. Die Ausdrücke $y_{2}, y_{3} \cdots y_{n}$ werden aber aus $y_{1}$ durch die Zusammenhangsgleichung von $y=f_{1}(x)$ hergeleitet, und da wir $y_{1}$ jeder Grösse gleich machen können, so folgt, dass $f_{1}^{\prime}(x)$ für solche Argumente denselben Werth annebmen muss, welche durch die Zusammenhangsgleichung von $y=f_{1}(x)$ in einander übergeführt werden können. Dies fällt aber mit der Forderung zusammen, dass $f_{-1}(x)$ denselben Zusammenhang wie $f_{1}(x)$ selbst besitzt. Wir haben daher nur solche functionen zu untersuchen, deren Zusammenhang mit dem ihrer Umkehrung übercinstimmt.

7. Die fragliche Function $y=f_{1}(x)$ möge durch die Gleichung 


$$
y^{n}+\varphi^{(1)}(x) y^{n-1}+\varphi^{(2)}(x) y^{n-2}+\cdots+\varphi^{(n)}(x)=0,
$$

ihre Umkehrung, welche nach dem Gesagten ebenfalls $n$-deutig ist, dureh

$$
y^{n}+\psi^{(1)}(x) y^{n-1}+\psi^{(2)}(x) y^{n-2}+\cdots+\psi^{(n)}(x)=0
$$

bestimmt sein, sodass die Gleichungen (1) und (2) durch Vertauschung von $y$ and $x$ in einander übergehen. Sollen beide Gleichungen denselben Zusammenhang haben, so muss das Eliminationsresultat von

$$
\sum y_{1} y_{2} \cdots y_{r}=\varphi^{(r)}(x)
$$

und

mit dem von

$$
\sum y_{1} y_{2} \cdots y_{s}=\varphi^{(s)}(x)
$$

$$
\sum y_{1} y_{2} \cdots y_{r}=\psi^{(r)}(x)
$$

und

$$
\sum y_{1} y_{2} \cdots y_{s}=\psi^{(s)}(x)
$$

ïbereinstimmen, d. h. es muss, wenn

$$
\psi^{(r)}(x)=\varphi^{(r)} \xi(x)
$$

ist, auch

$$
\psi^{(s)}(x)=\varphi^{(3)} \xi(x)
$$

sein. Es ergiebt sich dies leicht daraus, dass nach (3) und (4)

$$
\varphi^{(r)} \varphi_{-1}^{(s)}(x)=\psi^{(r)} \psi_{-1}^{(s)}(x)
$$

und zwar für alle Werthe dieser Ausdrücke sein muss, woraus bei der Annahme von (5) folgt

$$
\varphi^{(r)} \varphi_{-1}^{(s)}(x)=\varphi^{(r)} \xi \psi_{-1}^{(s)}(x),
$$

oder

$$
\varphi_{-1}^{(s)}(x)=\xi \psi_{-1}^{(s)}(x)
$$

oder endlich

$$
\psi^{(3)}(x)=\varphi^{(3)} \xi(x)
$$

Demnach kann (2) in die Form gesetzt werden

(11) $y^{n}+\varphi^{(1)} \xi(x) y^{n-1}+\varphi^{(2)} \xi(x) y^{n-2}+\cdots+\varphi^{(n)} \xi(x)=0$.

Dabei muss $\xi(x)$ so beschaffen sein, dass die Coefficienten eindeutige Functionen werden. Es folgt hieraus

$$
f_{-1}(x)=f_{1} \xi(x)
$$

Der letzteren Gleichung genügt aber nur

$$
\xi(x)=f_{-2}(x),
$$

und zwar ist klar, dass alle Werthe von $f_{1} f_{-2}(x)$ mit denen von $f_{-1}(x)$ übereinstimmen, weil nach Früherem beide Ausdrücke gerade $n$ Werthe 
haben und $f_{-1}(x)$ durch eine irreductible Gleichung bestimmt ist. Daher wird $\varphi^{(x)} f_{-2}(x)$ eine eindeutige Function sein müssen, was nur möglich ist, wenn alle $\varphi^{(x)}(x)$ vom, $n^{\text {(en }}$ Grad oder Constanten sind. $f_{-2}(x)$ hat mit der Umkehrung von $\varphi^{(x)}(x)$ denselben Zusammenhang, woraus weiter folgt, dass die Umkehrungen alter $\varphi^{(x)}(x)$, soweit diese nicht Constanten sind, von gleichem Zusammenhang sein, d. h. nach $\S 6,4$ durch folgende Gleichung bestimmt sein müssen:

$$
\frac{A_{0} y^{n}+A_{1} y^{n-1}+\cdots+A_{n}}{B_{0} y^{n}+B_{1} y^{n-1}+\cdots+B_{n}}=\frac{a x+b}{c x+d} .
$$

Die linke Seite dieser Gleichung ist bei allen $\varphi^{(x)}(x)$ dieselbe, während die rechte Seite nur vom ersten Grade zu sein braucht (da sonst die $\varphi^{*}(x)$ nicht rational wären), im Uebrigen aber verschieden sein kann. Die $\varphi^{(x)}(x)$ sind somit rationale Functionen des ersten Grades von derselben rationalen Function $n^{\text {ten }}$ Grades. Weiter ist noch ersichtlich, dass die Nenner der erstgenannten rationalen Functionen übereinstimmen müssen, da sonst beim Wegmultipliciren derselben die Gleichung (1) in $x$ von höherem als dem $n^{\text {ten }}$ Grade würde. Wir erhalten daher für (1)

oder

$$
\begin{aligned}
y^{n}+\frac{a_{1}-b_{1} \varphi_{1}(x)}{a_{0}-b_{0} \varphi_{1}(x)} y^{n-1} & +\frac{a_{2}-b_{2} \varphi_{1}(x)}{a_{0}-b_{0} \varphi_{1}(x)} y^{n-2}+\cdots \\
& +\frac{a_{n}-b_{n} \varphi_{1}(x)}{a_{0}-b_{0} \varphi_{1}(x)}=0
\end{aligned}
$$

$$
\frac{a_{0} y^{n}+a_{1} y^{n-1}+\cdots+a_{n}}{b_{0} y^{n}+b_{1} y^{n-1}+\cdots+b_{n}}=\frac{c_{0} x^{n}+c_{1} x^{n-1}+\cdots+c_{n}}{d_{0} x^{n}+d_{1} x^{n-1}+\cdots+d_{n}} .
$$

Durch nochmalige Anwendung von $\$ 6,4$, ergiebt sich aber hieraus leicht, dass $y=f_{1}(x)$ nur dann mit seiner Umkebrung denselben Zusammenhang haben kann, wenn (16) die Gestalt annimmt:

$$
\frac{a_{0} y^{n}+a_{1} y^{n-1}+\cdots+a_{n}}{b_{0} y^{n}+b_{1} y^{n-1}+\cdots+b_{n}}=\varepsilon\left(\frac{a_{0} x^{n}+a_{1} x^{n-1}+\cdots+a_{n}}{b_{0} x^{n}+b_{1} x^{n-1}+\cdots+b_{n}}\right),
$$

worin $\varepsilon$ eine lineare Function bezeichnet. Hiermit ist bewiesen, dass alle Periodicitätsgleichangen $n^{\text {ten }}$ Grades, welche iterirt immer vom $n^{\text {ien }}$ Grade bleiben, aus einer linearen durch Einsetzen einer rationalen Function $n^{\text {ten }}$ Grades für $y$ und $x$ hergeleitet werden können. Dass die gefundenen Gleichungen auch wirklich die gewünschte Eigenschaft besitzen, ist leicht ersichtlich*).

*) Das hier gefundene Resultat steht in vaher Beziehung zu dem von Herrn S. Lie in seiner "Theorie der Transformationsgrappen I", Mathematische Annalen, Bd. XVI, S. 455, ausgesprochenen; doch erkennt mall leicht, dass die beiden Sätze nicht identisch sind. Die von Herrn $\mathrm{S}$. Lie angewandten Methoden sind von den meinigen gänzlich verschieden. 
8. Es wird nicht ohne Interesse sein, wenn wir den eben allgemein bewiesenen Satz für einen speciellen Fall durch directe Rechnung herleiten. Sei die Funetion $y=f_{1}(x)$ durch die Gleichung

$$
a y^{2}+b y x+c x^{2}+d y+e x+f=0
$$

bestimmt, so werden wir die definirende Gleichung für die iterirte Function $z=f_{2}(x)$ finden, wenn wir $y$ aus der vorgelegten Gleichung und

$$
a z^{2}+b z y+c y^{2}+d z+e y+f=0
$$

eliminiren. Wir schreiben diese Gleichungen zuerst in der Form

$$
\begin{aligned}
& y^{2} a+y(b x+d)+c x^{2}+e x+f=0, \\
& y^{2} c+y(b z+c)+a z^{2}+d z+f=0,
\end{aligned}
$$

und erhalten dann nach einer bekannten Regel

$$
\left|\begin{array}{ll}
a & c x^{2}+e x+\left.f\right|^{2} \\
c & a z^{2}+d z+f
\end{array}\right|^{2}+\left|\begin{array}{cc}
c x^{2}+e x+f & b x+d \\
a z^{2}+d z+f & b z+e
\end{array}\right| \cdot\left|\begin{array}{ll}
a & b x+a \\
c & b z+e
\end{array}\right|=0 .
$$

Soll nun $z=f_{2}(x)$ eine höchstens zweideutige Function sein, so kann dies nur auf 2 Arten geschehen: entweder fallen in (20) die Potenzen von $x$ und $z$, welche den zweiten Grad überschreiten, weg, oder (20) lässt sich als Quadrat darstellen. Dass das Erstere nicht der Fall sein kann, ersieht man daraus, dass die Coefficienten von $z^{4}$ und $x^{4}, a^{4}$ und $c^{4}$ sind, die wir nicht beide als 0 annehmen dürfen. Das Letztere kann in 3 Fällen eintreten.

1. Ls ist für beliebige $x$ und $z$

$$
\left|\begin{array}{l}
c x^{2}+e x+f \quad b x+d \\
a z^{2}+d z+f, b z+e
\end{array}\right|=0
$$

oder

$$
\begin{aligned}
& b c x^{2} z+b e x z+b f z+c e x^{2}+e^{2} x+e f \\
= & a b x z^{2}+b d x z+b f x+a d z^{2}+d^{2} z+d f,
\end{aligned}
$$

eine Gleichung, die nur identisch befriedigt werden kann, wemn wir

$$
b=0, \quad d=0, \quad e=0
$$

annehmen. Die Gleichung

$$
a y^{2}+c x^{2}+f=0
$$

kann aber aus der inearen Gleichung

$$
a y+b x+f=0
$$

durch die Substitution $\psi_{1}(x)=x^{2}$ hergeleitet werden.

2. Es ist identisch

oder

$$
\left|\begin{array}{ll}
a & b x+d \\
c & b z+e
\end{array}\right|=0
$$

$$
a b z+a e=b c x+c a
$$


bier ist erforderlich, dass

$$
b=0 \text { und } a e=c d \text { oder } \frac{a}{c}=\frac{d}{e}
$$

ist; (18) nimmt dann die Form an

oder

$$
a y^{2}+d y+c x^{2}+\frac{c d}{a} x+f=0,
$$

$$
y^{2}+m y+p\left(x^{2}+m x\right)+f=0,
$$

eine Gleichung, die aus

durch die Substitution

$$
y+p x+f=0
$$

hervorgeht.

$$
\psi_{1}(x)=x^{2}+m x
$$

3. Fällt der zweite Theil von (20) nicht weg, so setzen wir zur Abkürzung

$$
\begin{aligned}
& r=b x+a, \\
& s=b z+e, \\
& u=c x^{2}+e x+f, \\
& v=a z^{2}+d z+f
\end{aligned}
$$

und erhalten durch Ausrechnung von (20)

$$
(a v-c u)^{2}-r s(a v+c u)+a s^{2} u+c r^{2} v=0,
$$

welcher Gleichung wir auch die beiden andern Formen geben können: (22) $(a v-c u)^{2}-r s(a v-c u)+a s^{2} u+c r^{2} v-2 c r s u=0$

und

(23) $(a v-c u)^{2}+r s(a v-c u)+a s^{2} u+c r^{2} v-2 a r s v=0$.

Ein Ausdruck $A^{2}+A B+C$ (dessen Bestandtheile $A, B, C$ verschiedene Variabeln enthalten) kaun aber nur dawn quadratisch sein, wenn $A B+C=0$ (die beiden vorigen Fälle) oder $B^{2}=4 C$ ist. Demnach haben wir wegen (22) und (23) die beiden Gleichungen zu befriedigen:

$$
r^{2} s^{2}=4 a s^{2} u+4 c r^{2} v-8 c r s u
$$

und

$$
r^{2} s^{2}=4 a s^{2} u+4 c r^{2} v-8 a r s v
$$

aus deren Zusammenstellung sich als nothwendige, doch nicht hinreichende Bedingung ergiebt

$$
\text { crsu }=a r s v .
$$

Diese Gleichung ist befriedigt, wenn $r=0$ oder $s=0$ ist; aus (24) und (25) folgt, dass dann auch die andere der beiden Grössen verschwinden muss (die übrigen Combinationen erweisen sich leicht als unzulässig); hieraus würde wieder wie oben $b=0, d=0, e=0$ 
folgeu. Andernfalls muss $c u=a v$ sein, was unmögliche Bedingungeu ergiebt.

Das Gesammtresultat lautet also: eine Function $y=\varphi_{1}(x)$, die durch eine Gleichung zweiten Grades definirt ist, giebt nur. dann wnd immer dann iterirt wieder cine Function zweiten Grades, wenn sic durch eine rationale Functionalsubstitution aus einer linearen Function hergeleitet ist.

9. Noch bleibt der Fall za betrachten übrig, dass die Iterirungen einer $n$-deutigen Periodicitätsfunction zwar mehr als $n$-deutig, aber so beschaffen sind, dass ein Theil ihrer Werthe mit solchen früherer Iterirungen zusammenfällt und in Folge dessen immer höchstens $n$ neue Werthe auftreten. Die erste Iterirung muss alsdann wieder $x$ als einen Werth haben, mithin muss nach Früherem die definirende Gleichung von $y=f_{1}(x)$ in $y$ und $x$ symmetrisch sein. Es ist mir noch nicht gelungen, diesen Fall zu erledigen, und ich beschränke mich darauf, durch ein Beispiel die vorkommenden Verhältnisse zu erläutern.

Sei

oder

$$
\sqrt{y}+\sqrt{x}=n
$$

$$
(y-x)^{2}-2 n^{2}(y+x)+n^{4}=0 .
$$

Diese Gleichung lässt sich durch die Functionalsubstitution

$$
\varphi_{1}(x)=\sqrt{x}-\frac{n}{2}
$$

aus

$$
y+x=0
$$

aber andrerseits auch in Folge der Doppeldeutigkeit der Wurzeln aus $y=x+n$ durch die Substitution $\varphi_{1}(x)=\sqrt{x}$ herleiten. Wir haben

$$
y=f_{1}(x)=(n \pm \sqrt{x})^{2},
$$

während wir bei der Iterirung den Werth $x$ doppelt und ausserdem

$$
f_{2}(x)=(2 n \pm \sqrt{x})^{2}
$$

finden. Für $f_{3}(x)$ erhalten wir wieder $(n \pm \sqrt{x})^{2},(2 n \pm \sqrt{x})^{2}$ und ausserdem $(3 n \pm \sqrt{x})^{2}$ u. s. w.

Dieser irrationalen Periodicitätsgleichung entspricht wirklich die eindeutige Function

$$
\cos \sqrt{x}=1-\frac{x}{1 \cdot 2}+\frac{x^{2}}{1 \cdot 2 \cdot 3 \cdot 4}-\frac{x^{3}}{1 \cdot 2 \cdot 3 \cdot 4 \cdot 5 \cdot 6},
$$

bei welcher

$$
y=(2 \pi \pm \sqrt{x})^{2}
$$

zu setzen ist. In der That ist

$$
\cos y=\cos (2 \pi \pm \sqrt{x})=\cos ( \pm \sqrt{x})=\cos \sqrt{x}
$$


In anderen Fällen gestalten sich die Verhältnisse freilich bedeutend anders; doch erscheint es nicht wahrscheinlich, dass sich periodische Functionen ergeben, welche nicht durch die Functionalsubstitution aus additiv oder multiplicatorisch periodischen hergeleitet werden können.

\section{$\S 8$.}

Die mehrfach periodischen Functionen.

1. Functionen mit mehreren (vorläufig 2) nicht auf einander zurückführbaren Perioden $\varphi_{1}(x)$ und $\chi_{1}(x)$ wollen wir zunächst nur für den Fall untersuchen, dass die letzteren vertauschbar sind, d. h. dass wir haben

$$
\varphi_{1} \chi_{1}(x)=\chi_{1} \varphi_{1}(x)
$$

auch beschränken wir uus im Folgenden auf rationale Periodicitätsgleichungen.

2. Die eine der Perioden können wir immer in eine adclitive oder multiplicatorische transformiren; dic directe Rechunug ergiebt danu ohne Schwierigkeit, dass die andere jedesmal von derselben Art sein muss; es kommen daher für uns nur Functionen mit mehrfacher arlditiver oder mehrfacher multiplicatorischer Periode in Betracht.

Was den ersten Fall anlangt, so ist bekannt, dass die Zahl der additiven Perioden (bei endlich vieldeutigen Functionen) 2 nicht überschreiten kann und dass zwischen diesen beiden Perioden kein reelles Verhältniss bestehen darf.

3. Jede eindeutige (oder endlich vieldeutige) Function mit multi- plicatorischer Periode lässt sich in eine eindentige (resp. ebensovieldeutige) Function mit doppelter additiver Periode mittelst einer transcendenten Substitution verwandeln. Denn setzt man in der Function

$$
\begin{gathered}
F(p x)=F(x) \\
p=e^{\frac{2 \pi i m}{n}}, \quad x=e^{\frac{2 \pi i w}{n}},
\end{gathered}
$$

so hat $F\left(e^{\frac{2 \pi i w}{n}}\right)$ die doppelte Periode $m$ und $n$. Ist ferner

und

$$
F_{1}(w+m)=F_{1}(w)
$$

so substituiren wir

$$
F_{1}(w+n)=F_{1}(w)
$$

$$
w=\frac{n}{2 \pi i} l x
$$

und gelangen hierdurch zu einer multiplicatorisch periodischen Function. Die unendliche Vieldeutigkeit von $l x$ macht die Function nicht vieldeutig, da die verschiedenen Werthe von $w$ die Form haben $w=\frac{n}{2 \pi i} l x+k n$ und durch die lufügung von $k n$ zu $w$ die Func- 
tion $F_{1}(w)$ nicht geändert wird. - Hieraus folgt auch leicht, dass mod. $p$ von der Einheit verschieden sein muss.

4. Machte man bei einer Function mit doppelter multiplicatorischer Periode die eben erwähnte Substitution, so würde man eine Function mit dreifacher additiver Periode erhalten; endlich vieldeutige Functionen mit mehrfacher multiplicatorischer Periode sind also nicht möglich, wenn wir selbstverständlich den Fall ausschliessen, dass die lerioden ganze Potenzen derselben Grösse sind.

Wir haben somit nur 3 Arten von periodischen trauscendenteu Functionen gefunden :

1. die Functionen mit einfacher additiver Periode,

2. diejenigen mit cinfacher multiplicatorischer Periode,

3. diejenigen mit doppelter additiver Periode.

Die letzteren braucht man jedoch nicht besonders zu behandeln, weil sie sich aus den vorhergehenden herleiten lassen. Diese Reduction der elliptischen Functionen auf die Modularfunctionen ist von grossem Vortheil, da die letzteren einfacher sind und hierdurch ihre analytische Natur deutlicher hervortreten lassen.

5. Bei mehrfachen Perioden, für welche die Gleichung

$$
\varphi_{1} \chi_{1}(x)=\chi_{1} \varphi_{1}(x)
$$

nicht gilt, hört die Analogie mit den Abel'schen Gleichungen auf; denn A bel hat die algebraische Lösbarkeit solcher Gleichungen, deren Wurzeln durch die Iterirungen von 2 verschiedenen Functionen aus einer einzigen hergeleitet werden können, nur unter der Voraussetzung bewiesen, dass diese beiden Functionen vertauschbar sind. Ueberdies ist es walarscheinlich, dass mehrere nicht vertauschbare Perioden (einzelne Specialfälle ausgenommen) bei Functionen mit getrennt liegenden Discontinuitätspunliten nicht möglich sind; vorläufig gelang es mir nur nachzuweisen, dass Functionen mit zwei nicht vertauschbaren Perioden in Allgemeinen unendlich viele wesentliche Discontinuitätspunkte besitzen müssen. Manche Combinationen von Perioden sind bei eindeutígen Functionen überhaupt nicht zulässig, wie aus der Untersuchung des Specialfalles, dass sich beide Periodicitätsgleichungen durch Transformation gleichzeitig von einem Gliede mit $x y$ befreien lassen, hervorgehen wird.

a) Die eine Periodicitätsgleichung lasse sich auf

$$
\varphi_{1}(x)=x+1
$$

reduciren, während die andere hierbei in

übergeht. Dann ist

$$
\chi_{\mathrm{t}}(x)=a x+b
$$

$$
\begin{aligned}
\chi_{n}(x) & =a^{n} x+b\left(a^{n-1}+a^{n-2}+\cdots+a+1\right), \\
\chi_{-n}(x) & =\frac{x}{a^{n}}-b\left(\frac{1}{a^{n}}+\frac{1}{a^{n-1}}+\cdots+\frac{1}{a}\right),
\end{aligned}
$$


also

(5) $\quad \varphi_{1} \chi_{n}(x)=a^{n} x+b\left(a^{n-1}+a^{n-2}+\cdots+a+1\right)+1$,

(6) $\quad x_{n} \varphi_{1}(x)=a^{n} x+b\left(a^{n-1}+a^{n-2}+\cdots+a+1\right)+a^{n}$,

und somit

$$
\begin{aligned}
& F\left[a^{n} x+b\left(a^{n-1}+a^{n-2}+\cdots+a+1\right)+1\right] \\
= & F\left[a^{n} x+b\left(a^{n-1}+a^{n-2}+\cdots+a+1\right)+a^{n}\right],
\end{aligned}
$$

oder weln $x$ für $a^{n} x+b\left(a^{n-1}+a^{n-2}+\cdots+a+1\right)+1$ gesetzt wird,

oder wegen (1)

$$
F\left(x+a^{n}-1\right)=F(x)
$$

$$
F\left(x+a^{n}\right)=F(x),
$$

ein Resultat, das auch für negative $n$ gilt, wie aus (4) folgt. Ist mod. $a$ von der Einheit verschieden, so kann $a^{n}$ durch wachsende positive oder negative $n$ beliebig klein gemacht werden; $F(x)$ müsste also eine unendlich kleine additive Periode haben, was bei endlich vieldeutigen Functionen nicht möglich ist. Wenn mod. $a=1$ ist, so kann zunächst $a$ genau eine Einheitswurzel sein; dann ist $\chi_{1}(x)=a x+b$ eine Periode, die durch Iterirung zu $x$ zurückführt, wie aus Früheren sowohl als auch aus (3) erhellt. Ist nänlich $a$ eine primitive $n^{\text {te }}$ Einheitswurzel, so haben wir

also

$$
\begin{gathered}
a^{n}=1, \\
a^{n-1}+a^{n-2}+\cdots+a+1=0,
\end{gathered}
$$

$$
\chi_{n}(x)=x \text {. }
$$

Trifft diese Voraussetzung nicht zu, so lässt sich leicht nachweisen, dass unendlich viele unendlich wenig von einander verschiedene additive Perioden vorhanden sind, indem $a^{n}$ durch geeignete Wahl von $n$ jeder Grösse vom Modul 1 beliebig nahe gebracht werden kann. - Für $a=1$ ist auch die zweite Periode additiv.

b) Sei die eine Periodicitätsgleichung auf

reducirbar, während die andere wieder

$$
\varphi_{1}(x)=p x
$$

$$
\chi_{1}(x)=a x+b
$$

lauten möge ( $b$ von Null, mod. $p$ von 1 verschieden), so ist

$$
\begin{aligned}
& \varphi_{1} \chi_{1}(x)=a p x+b p, \\
& \chi_{1} \varphi_{1}(x)=a p x+b,
\end{aligned}
$$

woraus wie oben folgt

$$
F[x+b(p-1)]=F(x),
$$

wodurch dieser Fall auf den vorhergehenden zurückgeführt ist. 
6. Ist

$$
\begin{aligned}
& \varphi_{1}(x)=x+1, \\
& \chi_{1}(x)=\frac{a x+b}{c x+d},
\end{aligned}
$$

so muss wegen (14) die Function für $x=\infty$ wesentlich discontinuirlich werden; aus (15) folgt dann, dass dies auch für $x=\frac{a}{c}$, also wegen beider Gleichungen in unendlich vielen Punkten geschehen muss. Ein Ausnahmefall ist nicht möglich, wenn $c$ von Null verschieden ist. Tritt an Stelle ron (14)

$$
\varphi_{1}(x)=p x,
$$

so ist eine analoge Betrachtung möglich; auch hier müssen unendlich viele wesentliche Discontinuitätspunkte vorhanden sein, wenn nicht

oder

$$
\chi_{1}(x)=q x
$$

$$
\chi_{1}(x)=\frac{q}{x}
$$

ist; im letzteren Fall ist

$$
\chi_{2}(x)=x
$$

Frankfurt a/M., im Februar 1881. 